\title{
Almond (Prunus dulcis): A Nutritive Dense Dry Fruit
}

\section{Waseem Khalid ${ }^{1 *}$, Fareed Afzal ${ }^{1}$, Ravi Prakash Jha ${ }^{2}$, Nageen Afzal ${ }^{1}$, Muhammad Zubair Khalid ${ }^{1}$, Talal Shoaib ${ }^{3}$, Ramish Akram ${ }^{4}$, Poonam Gill $^{5}$, Afifa Aziz ${ }^{1}$, Noman Aslam ${ }^{1}$ and Areeg Azhar ${ }^{1}$}

${ }^{1}$ Department of Food Sciences, Government College University, Faisalabad, Pakistan

${ }^{2}$ Department of Community Medicine, Institute of Medical Sciences, Banaras Hindu University, Varanasi, India

${ }^{3}$ Center for Agricultural Biochemistry and Biotechnology, University of Agriculture, Faisalabad, Pakistan

${ }^{4}$ Department of Rehabilitation Sciences, The University of Faisalabad, Pakistan

${ }^{5}$ National Institute of Food Science and Technology, University of Agricultural

Faisalabad, Pakistan

*Corresponding Author: Waseem Khalid, Department of Food Sciences, Government

College University Faisalabad, Pakistan. E-mail: waseemkhalid@gcuf.edu.pk
Received: May 21, 2021

Published: June 11, 2021

(C) All rights are reserved by Waseem Khalid., et al.

\section{Abstract}

Almond belongs to plant-based food and is botanically called Prunus dulcis. The edible part of the almond is the seed that is composed of different nutritive and non-nutritive components. Almond seed consists of fat, protein, carbohydrates, vitamins, minerals, antioxidants and phenolic compounds. These compositional constituents of almond seed play important role in the human body. It is helpful in heart disease and diabetes because it is a good source of antioxidants and polyphenols that prevents inflammation of cells. Almond seed fiber is suitable for preventing constipation. Conclusively, its seeds are composed of many beneficial components that can reduce the chances of diseases.

Keywords: Almond; Vitamins; Minerals; Phenolic Compounds; Health Benefits

\section{Introduction}

Almonds which belongs to the Rosacea family and many other belongs to this family like raspberries, prunes, pears and, apples, are one the famous tree nuts around the globe and remain on the top among all of the tree nuts production all over the world. Almonds are mostly used by the snacks food industries and it is also part of bakery industries as well as confectionery industries [1]. The sweet almond belongs to the family called Rosacea. Almond is a drupel of which the special part is the nut seed or nut kernel
[2]. The latter is formed of an embryo, enclosed by skin also called testa. The pericarp, which embeds the seed, carries a green fleshy hull and a solid-pitted shell. The United States of America is the biggest producer of almonds. California is the largest producer of almonds in the United States of America as well as around the world). Almost 400 miles covered with almonds orchards in California [3]. Approximately 400,000 acres were cultivated by more than 7000 producers. California's biggest fruit nut crop is almond which crop is based on high-income value and cultivated in a large area and its impotence by worldwide distribution. Nonpareil, Mission, Califor- 
nia, Neplus Ultra, and Peerless are major classes of almonds grown by a cultivator in California. In market contribution, if we count by percentage then almost $90 \%$ contribution comes from these types Mission, Nonpareil, and California are fruit nut seeds of almond [1].

Almonds fruit consists of three parts $1^{\text {st }}$ - Kernel/Meat $2^{\text {nd }}$-Shell $3^{\text {rd }}$-Hull. There are two types of almonds based on texture Soft shell and hard shell. Almond shells start drying on the trees its points that they are ready to $b$ harvest [4]. In addition, sweet almonds have many other features to be stone fruit. It is unique among in his botanical family-like plum, apricot and peach, where the flesh (mesocarp) of the particular fruit is eaten and the seed within the shell is to be discarded, the converse is $100 \%$ true for almond during its ripening cycle, for a period few days to few weeks to become a fully ripe fruit (seed, endocarp and mesocarp). With time when almond ripened fruit enter in maturation period cycle, the hull bursts open. When it's dried it is easy to separate the hull from the almond shell. The almond shell inner pit/seed is used for human consumption, nut for trade purposes, the shell and hull is used for animal feeds and cat litter. Commercially large exporters or producers are mainly in Australia, the USA, Iran, Turkey, Morocco, Spain and, Pakistan $[5]$.

Shell or Unshelled almonds are sold in every corner of the world as whole natural almonds or maybe processed almonds forms. The shell is likely to be a throw-away but its brown skin is to be preserved for further usage: but in the case of blanched almonds both their shell and skin are discarded. Generally, the skin of almonds was discarded [1]. Nuts and edible seeds are a good source of macronutrients and micronutrients. That type of food also contains bioactive and antioxidants compounds which reduce the risk of cardiovascular issues and chronic problems in the human body [6]. Most of the nuts have a higher lipid profile and high nutritional base value. If we take a little amount of fats for the nuts source so we can minimize the risk of LDL Cholesterol more than half in the human body [7].

The physiological benefits of bioactive compounds present in nuts maintain lipid-altering activities just because of their synergistic effects with each other. After eating nuts, we get antioxidants which are responsible to protect the human body by lowing the oxygen mass, it stops the chain which is responsible for hydrogen removal from the substance, decomposing of the first stage of oxidation to non-radical, stops the initial chain reaction by hunting down the free radicals, stops the process of singlet oxygen making, ion catalysts bonded by them [8]. Almonds when added to the daily base diet is reported that's it reduce the factors that cause colon cancer in the large intestine in rats [9] and its raise the good cholesterol level and decrease the level of bad cholesterol level in the human body [10].

Acceptance of edible nuts around the globe, because of their unique health-promoting properties, good taste, and especially sensory characteristics which make them unique in all kinds of fruits [11]. Almond, very well known as Prunus dulcis, belongs to the family name is Rosacea, Almonds production is on the top in the ranking of nuts production around the world [8]. Mainly it is cultivated in the Mediterranean region due to its unique climate and fertile lands. This region is responsible to fulfill the $28 \%$ food requirements. Almond tree has great potential to make money or high economic value [12]. There are so many different types of almonds that manifest different yields of edible seed in the fruit and fertility [13]. In confectionery and baking industries almonds are used with or without skin or as a whole nut [8]. There is a very large amount of bioactive and phytochemicals and antioxidants are present in nuts along with sugars or other polyols class via O-glycoside bonds or ester bonds [14] and chronic diseases can be controlled by eating a moderate amount of almonds [15]. The Prunus genus is satisfying to have very unique biological properties such as sedative, antioxidant activities, anti-carcinogenic, antiinflammatory [16].

A brown leathery covering on the body of almond is called the seed coat, which protects against microbial decaying and oxidation. Many other uses of almond are in confectionery, bakery, cereals, and marzipan in the formulations of snacks [17]. Almond skins are a farming by-product that is generated by the processing of a massive amount of almond is an origin of phenolic composites. Almond's brown skin, generally produced through the hot water blanching process, is milled and use as a cattle's feed or burned as fuel in-plant processing [18]. The brown skin of almond is almost $4 \%$ of the total fruit of almond and was merely a reachable source of phenolic and flavonoids [19]. These flavonoids and phenolic compounds are responsible to hinder the fats and oils oxidation by trapping the free radicals and inhibits the enzymes, activating antioxidant enzymes, by lowing the tocopherol radicals and also inhibit the growth of those enzymes which cause oxidation reac- 
tions [20]. Still, 21st-century studies have proven that the combination of phytochemicals in almond skins that the seed-coat may include possibly beneficial composites, it's creating new ways for value addition of almond's seed-coat [21]. In the almond's seed layer several phenolic syntheses have been recognized for separate Flavonol glycosides, commonly kaempferol glycoside, kaempferol rutinoside, isorhamnetin glucoside and, isorhamnetin rutinoside have been recognized in the seed-coat of almond [8]. Besides this Scientist has been found phenolic composites in the brown skin of almond, for example, a benzoic acid derivative, vanillic acid, protocatechuic acid, catechin, naringenin, kaempferol, galactose, glucose, and quercetin to glycosylated [22]. In extension, the phenolic mixture's classification and, separation have been made by [23].

Almond shells have a good amount of xylan contents which is better subtract for the xylose product [24], Presence of furfural is also found by [25] lignin, cellulose and pentosans fractionation. After a longer period, they found acid-catalyzed hydrolysis but this was is late utilization of hulls, as well as this starts from solubilisation and depolymersation of the principal elements which occurs in hemicelluloses. Acetic acid and aggregates are obtained from the acid-soluble lignin when the liquid phase (hydrolysate) comprises the sugars and sugar dehydration. Further utilization for oxyaromatics which are beneficial for the human health, development of cosmetics and also been used in food products [25]. The presence of lignins on the curst of almond is very high (30-38\% of the total dry weight) [26]. Syringyl phenylpropane to guaiacyl unit's ratio is similar to that of solid woods [25]. Most of the lignin is acid insoluble; a particular portion may be solubilized in media that contain acids. The free radical scavenging activity of de polymerized lignin fragments conveys after slightly acid hydrolysis of lingo cellulosic has been stated [27].

\section{Functional components in almond}

\section{Antioxidant potential of almonds}

Several considerations have been centered on the protecting and defensive impact of an antioxidant action on certain degenerative diseases such as cardiovascular, cancer, neurological disorders, cataracts, and oxidative tension malfunction. The existing work was carried out to assess the antioxidant action of prepared dehydrated fruits. An entire antioxidant activity was evaluated by the decreasing power and superoxide anion scavenging action assays. The consequences of decreasing energy and scavenging ability revealed that the reducing power increases with the density in two processing techniques (roasting and boiling with milk) but drops in the other two processing mean (long and shallow frying). The superoxide anion scavenging capacity showed that frying (deep and shallow) changes higher to the antioxidant action while the other two processing (roasting and boiling with milk) had limited influence on their antioxidant effectiveness [28].

Fatty acids profile of almonds

Classifications of fatty acids contents in \% of total methyl ester of fatty acids (FAMEs) of 14 vegetable oils, grape, safflower, hemp, Silybum marianum, wheat germ, sunflower, pumpkin seed, almond, sesame, rice bran, olive, coconut oil, rapeseed, and peanut, were obtained by using (GS) Gas chromatography. Polyunsaturated fatty acids (PUFA), monounsaturated (MUFA), Saturated (SFA) and monounsaturated (MUFA), oleic acid (C18:1; 6.2\% - 71.1\%) and linoleic acid (C18:2; 1.6\% - 79\%), palmitic acid (C16:0; 4.6\% - 20.0\%), commonly, were found main fatty acids. The nutritional feature of evaluated oils was analyzed by determination of the energy contribution of PUFAs (10.6\%-786.8\% ERDI), n-3 FAs (4.4\% - 117.1\% ERDI), n-6 FAs (1.8\% - 959.2\% ERDI), and SFAs (19.4\% - 695.7\% ERDI) expressed in \% ERDI of One-gram oil to energy recommended dietary intakes (ERDI) for total fat (ERDI-37.7 kJ/g). The important relationship between the reported data of total fat, PUFAs MUFAs, and SFAs, intakes (\% energy recommended dietary intakes) for adults, and mortality caused by cardiovascular diseases (CVD) and coronary heart diseases (CHD) in 12 countries have not been confirmed by Spearman's correlations [29].

\section{Phenolic compounds}

There is a wide range of antioxidants, bioactive and phytochemicals present in almond-like phenolic, flavonoids, pro anthocyanidins [30]. Almond brown's skin is the origin of these phenolic and flavonoids where these compounds found in large amount and very known for their antioxidant potential [31]. Phytosterols play an important in the human body (270 mg 100g1) found in almonds, b-sitosterol being the predominant variety [32]. Phytosterols help to maintain blood concentration of LDL cholesterol and also these composites provide help to maintain heart health in the human body after consuming almonds [33]. A Very low amount of amygdaline ( 0.2 to $16 \mathrm{mg} / 100 \mathrm{~g}$ of almond) is present in sweet almonds. Toxic cyanogenic glycoside, whereas bitter almonds have great level of this glycoside (3300 to $5400 \mathrm{mg} / 100 \mathrm{~g}$ ) [34]. 
There is great potential for free radical scavenging capacities in Almond Shell, Kernel and, brown skin [35]. Flavonoids and other phenolic composites are also present in almond nuts. Almond byproduct Hulls have been observed to show the great potential of three triterpenoids (almost 1\% of the hulls) oleanolic acids, betulinic and ursolic [36]. Flavonol glycosides and phenolic acids are also present in hulls [1], separated catechins, naringenin glucoside, protocatechuic acid, p-hydroxybenzoic acid and vanillic acid and also galactoside, glucoside, and rahmno glucoside of kaempferol and rhamno glucoside of $3 \mathrm{~b}-\mathrm{O}$ methyl quercetin. The by-product of almonds used in feed to animals is an approximately 5443108.44 metric tons produced that likely to a great source of bioactive and antioxidant compounds present in hulls [37].

\section{Health benefits of almond}

It is imagined that almond is wealthy as far as vitamin E, copper, phosphor, manganese, magnesium, potassium, fiber, riboflavin, monounsaturated unsaturated fats, and protein. As a result of its one of a kind composites of cancer prevention agents so as isorhamnetin, quercitrin, kaempferol, quercetin, catechin, epicatechin, naringenin, cyanidin, delphinidin, 3-0-rutinoside, isorhamnetin 3-0-glucoside, morin, caffeic corrosive, ferulic corrosive, p-coumaric corrosive, protocatechuic corrosive and vanillic corrosive, it is said that almond is in a unique position as far as human nourishment. Cell reinforcements have an imperative effect on DNA's misfortune restraint as counteracting LDL cholesterol oxidation. In various investigates which have been learned about almond's effects on human wellbeing, the fat and unsaturated fats creation of almond's species has been found and it has been demonstrated that almond: is wealthy for monounsaturated unsaturated fats, it has oleic corrosive which is one of unsaturated fat that has the motivation behind diminishing cholesterol level in the blood, and it has linoleic corrosive which restrains intravenous impediment. Almond is the best birthplace among different nuts regarding vitamin $E$ which is recognized as the solvent lipid phenolic cancer prevention agent. It has been demonstrated that vitamin $\mathrm{E}$ has a basic part keeping in mind the end goal to stop diabetes, atherosclerotic vascular issues, and some growth infections.

Moreover, almonds have a low glycemic index and do not unfavorably affect insulin responsiveness. While the advantages of almonds for cardiovascular health and obesity-related conditions appear promising, the possible allergenic response among sensitive individuals can present a danger. L-Arginine in almond has been proved to be a powerful negotiator of multiple biological means, including the discharge of several hormones, collagen synthesis while wound healing, antitumor action, and immune cell answers. Almond oil is practiced today by massage therapists for its many natural emollient and skin-rejuvenating characteristics. Further, it may have a useful influence on the management of sensitive bowel syndrome. Also, almond is a good origin of mineral compounds especially: calcium, magnesium, phosphorus, and potassium. These syntheses have significant augmentations to human health in terms of supporting hypertension, including the bone and teeth, blood-forming, nerve stimulation, growth, and gender development including muscular tissue activity. Researches which have been studied on almond's nutritional aspects, maintain the approach that almond is a useful nutrient that has a medical advantage on the therapy of some disorders [38].

\section{Weight maintenance and type 2 diabetes}

Health studies recommended that those who eat stone fruit periodically ( 5 times a week) contribute to having lower body mass indices [39]. These calculations led to exploration on stone fruit to find out possible mechanisms of weight loss and weight maintenance. Almonds are good in protein and fiber and have a little glycemic index, all of which are dietary aspects shown to suppress appetite and increase satiety [40].

In 2008, the energetics of stone fruit consumption was reviewed by Mattes, and 3 possible mechanisms were expected the $1^{\text {st }}$ spotlight on the satiety value of stone fruit, and it is hypothesized that the formation of stone fruit in the diet results in a spontaneous depletion of energy absorption at other times of the day to balance a high portion of the energy contributed by the nuts. $2^{\text {nd }}$, it is suggested that nut consumption may boost energy levels and thereby dissipate a part of the energy they contribute. $3^{\text {rd }}$, it is recommended that the consumption of the energy from nuts is debilitated, thereby reducing their theoretical addition to energy intake Utilizing almonds being a model [41].

Tried to quantify the energetics of nut used in a randomized crossover, experiment that was involved 20 healthy adult female cases with a body mass index (BMI) of $25.93 .1 \mathrm{~kg} / \mathrm{m}^{2}$. Two 10 week analysis periods were distributed by a three-week washout interval. Meanwhile, one arm of the study, $1440 \mathrm{~kJ} /$ day of almonds 
was given with no dietary advice besides that the day's portion of almonds had to be eaten. A second arm changed only in that nuts were forbidden. The results unveiled the strong satiating results of almonds, with $74 \%$ of the energy from almonds offset by decreased energy intake from other origins. There was a notable increase in fecal energy waste, considering about $7 \%$ of the energy of almonds and a non-significant rise in daily energy expenditure that would consider from approximately $14 \%$ of the energy of almonds. Therefore, the conclusions exhibited the least influence of almond using on body weight. These tools help reveal the consequences of epidemiological and clinical studies which recommend that average nut consumption does not act as a threat to the weight increase. For illustration, a southern Californian study revealed that adding a modest quantity of almonds ( $65 \mathrm{~g}$ ) to the diet for six months resulted in no important changes in body weight and an expansion in the balance of unsaturated fat in the diet for 81 cases [42].

The authors stated that food displacement happened later almond supplementation, and over $54-78 \%$ of additional calories from almonds were removed by a reduction in consumption of other few healthy foods in the conventional diet [43]. A reasonable fat diet with almonds produced more weight loss than a lowfat diet, even though the total number of calories in the six-month study duration was the same for both groups. In extension, the almond group had a 50\% greater reduction in waist limit and a $62 \%$ greater decrease in fat mass than the low-fat diet group. Replacing almonds with other foods in the diet that are not as satiating is a potential strategy for weight loss and weight sustaining. Almonds can restore less nutrient-dense foods in the diet and eating extra nutrient-dense foods includes fewer calories to fulfill nutrient conditions. Data concerning the long-term relationship between nut using and weight shifts in a free-living population are rare [44].

Brought out and considered study of nut using, long-term weight development, and obesity chance in women. Greater nut consumption was not correlated with elevated body weight gain during 8 years of follow-up in healthy middle-aged women. Instead, it was associated with a slightly lower risk of weight gain and obesity. The authors decided that the inclusion of nuts into habitual diets does not lead to greater weight increase and may offer weight control. With the constant rise in the prevalence of obesity, it is becoming extra valuable for scientists and health professionals to recognize the use of nuts in body weight control and the associated chronic diseases. Analysis of the available data implies that supplementing nuts to regular diets of free-living people does not create weight increases. In particular, the indication so far shows that nuts have a trend to lower body weight and fat mass [45].

Data from epidemiological and human interference studies are facing a protecting role for nuts, and almonds in special, in developments in glycemic administration, insulin responsiveness, and lessening risk factors for diabetes [46]. Although there is some proof to recommend that almonds, and possibly other nuts, may have a positive effect on insulin consciousness, further studies are required to explain the potential tools. Diverse studies have shown that the chance of type 2 diabetes is reduced with greater intakes of dietary fiber and lower glycemic amounts. Jenkins and colleagues lately assessed the efficacy of either mixed nuts or almonds to change glucose spikes that befall after eating carbohydrate-rich foods that generally increase blood sugar levels [47]. The conclusions pointed that in healthy men and women, consuming nuts with a carbohydrate-rich meal blunted the glycemic and insulin response of the body to an important degree [46].

\section{Effect of almond on total cholesterol}

Monosodium glutamate (MSG) implies a synthetic aggregate that when it is practiced extremely it can cause several side consequences such as hypercholesterolemia. Hypercholesterolemia will rise in coronary heart illness (CHD), which is a condition beginning death. The object of the study was to evaluate the impact of almond nut/Prunus dulcis infusion versus total cholesterol level in mice that were given MSG. This research was a randomized posttest controlled organization design. The sample consisted of 30 mice (Mus musculus L. Strain DDW) that were classified into three groups: control group, treatment group 1, and treatment group 2 . The plasma entire cholesterol of mice was estimated by the means of CHOD with a spectrophotometer whose wavelength $500 \mathrm{~nm}$. Data recovered was analyzed by SPSS program version 16 . The base complete cholesterol level in mice that were employed with Prunus dulcis extraction later 4 weeks trial was $130.37 \mathrm{mg} / \mathrm{dl}$. It was highest related to the other groups. One-way ANOVA showed the importance rate was $p=0.44$ ), which suggests, there was no variation of complete cholesterol level among control and therapy groups 1 and 2. There was no impact of Prunus dulcis extract facing entire cholesterol level into mice that given monosodium glutamate [48]. 
Health point of view major cardiovascular diseases

Almonds supply a rich-nutrient source of, manganese, vitamin E, magnesium, phosphorus, copper, riboflavin, and fiber, protein, and monounsaturated fatty acids. Although about fifty percent of almond weight is fat, additional intakes of $7 \mathrm{~g}$ day- 1 of this stone fruit reduce (LDL) cholesterol concentration by 1\%, particularly within the discourse of diets suggested by the National Cholesterol Education Program. Usual almond eating does not lead to obesity, and their incorporation in low-calorie diets appears to help more weight loss than an equal carbohydrate-based low-calorie diet. As well as, almonds have a low glycemic index and do not adversely contact insulin sensitivity. Almonds are a good source of bioavailable $\alpha$-tocopherol and increasing their intake upgrades the resistance of Low-density Lipoproteins against oxidation. As well as, the polyphenolic components of almonds have been identified newly and found to maintain antioxidant activities. While the assistance of almonds for obesity and Heart-Health associated disorders come out promising, the possible allergenic attitude among sensitive individuals can present a Danger. Further research is needed to achieve an improved understanding of the role that the bioaccessibility and bioavailability of almond constituents and the synergy amid them play in their associated health-related result [49].

\section{Almond oil for skin}

Almond oil originates from Oleum amygdalae. Almond oil has emollient and sclerosant characteristics, which have been practiced to enhance pigmentation and skin tone. A nonrandomized comparison (50) has confirmed that massage with bitter almond oil may be effective in reducing the clarity of modern striae gravidarum, and in the inhibition of new striae [50].

Extra formulations have been shown to ameliorate striae itching [51] Nevertheless, other results including almond oil have not proved to have a comparable benefit [52]. For example, sweet almond oil in creams is more efficient than the base cream at ameliorating the itching of striae and limiting its sequence [51]. In a comparison by Sultana et al. done with murine models, topical almond oil was proved to limit the fundamental damage induced by UV irradiation [53].

\section{Almond reduced risk of cardiovascular disease}

Epidemiological investigations have been especially constant in determining a relationship between nut eating and a lessened chance of CHD [54]. All present a clear dose-response gradient within nut using and decreased CHD risk. Practiced collectively, these observational comparisons showed an ordinary chance decline of CHD death of $37 \%$ (relative risk (RR) 0.63 ; 95\% certainty interval (CI): $0.51,0.83$ ) or a percentage of $8.3 \%$ contraction in danger of CHD death for each weekly portion of nuts [55].

The advantageous effects of nut using are related to several clinical results: nonfatal myocardial infarction, fatal CHD, and sudden cardiac mortality. Collectively, these epidemiological conclusions give clear proof of the cardio-protective advantages of nut using [54]. The low prevalence of CHD in Mediterranean countries has been slightly ascribed to dietary practices, and modern verdicts from large European cohort investigations [56] imply that a great degree of adherence to the old Mediterranean diet is compared with a decrease in fatality [57]. Tree nuts as mentioned before almonds, hazelnuts, and walnuts, which are popular in the Mediterranean food, have advantageous fatty acid forms and are a rich reservoir of nutrients and other bioactive aggregates, which may consider in part for the noted advantageous consequences on the decreased chance of CHD [58].

Members were allotted to a low-fat food (n 1/4 257) or on one of two Mediterranean foods. Those allocated to Mediterranean menus experienced nutrition literacy and both free virgin olive oil (1 L/week (n 1/4 257)) or tree nuts (15 g/day walnuts, $7.5 \mathrm{~g} /$ day hazelnuts, and $7.5 \mathrm{~g} /$ day almonds, for 3 months (n $1 / 4$ 258)). The finish rate was $99.6 \%$. Associated with the low-fat diet, the two Mediterranean foods enriched with olive oil or nuts provided advantageous effects on cardiovascular danger circumstances, including blood glucose, systolic blood pressure, and complete cholesterol to high-density lipoprotein (HDL) ratio [59].

These authors achieved that a Mediterranean intake including nuts such as almonds could be a valuable means in training individuals who are in great jeopardy of cardiovascular disorder. Likewise, a Mediterranean-style fare with comparatively greater consumption of nuts and legumes and high in MUFA was determined to improve glucose metabolism more than a typical Western diet [60]. In conclusion, frequent nut consumption reduces the risk of cardiovascular disease, a result that has been recognized in different populations and is self-governing of other lifestyle circumstances [55]. Nut eaters typically consume inadequate meat, have 
lower consumption of trans fatty acids and higher consumptions of unsaturated fatty acids including fiber [46].

\section{Conclusion}

In recent review concluded that almond seed is also called dry fruit belongs to the plant family. Its seed is a rich source of bioactive compounds including antioxidants, phenolic compounds, vitamins and minerals. These compounds are considered beneficial in chronic diseases such as cardiovascular disease, diabetes and chronic constipation. In the future, further study required to design different products in which almond will be used as a functional ingredient.

\section{Bibliography}

1. Sang Shengmin., et al. "New prenylated benzoic acid and other constituents from almond hulls (Prunus amygdalus Batsch)". Journal of Agricultural and Food Chemistry 50.3 (2002): 607-609.

2. Gradziel Thomas M. "Redomesticating almond to meet emerging food safety needs". Frontiers in Plant Science 11 (2020).

3. Sathe Shridhar K., et al. "Biochemical characterization of amandin, the major storage protein in almond (Prunus dulcis L.)". Journal of Agricultural and Food Chemistry 50.15 (2002): 4333-4341.

4. King A Douglas., et al. "Almond harvesting, processing, and microbial flora". Applied Microbiology 20.2 (1970): 208-214.

5. Rabinowitz I N., et al. U.S. Patent No. US132,031. Washington, DC: U.S. Patent and Trademark Office (2002).

6. Freitas Jullyana Borges and Maria Margareth Veloso Naves. “Composição química de nozes e sementes comestíveis e sua relação com a nutrição e saúde". Revista de Nutrição 23.2 (2010): 269-279.

7. Abbey Mavis., et al. "Partial replacement of saturated fatty acids with almonds or walnuts lowers total plasma cholesterol and low-density-lipoprotein cholesterol". The American Journal of Clinical Nutrition 59.5 (1994): 995-999.

8. Wijeratne Subhashinee SK., et al. "Antioxidant activity of almonds and their by-products in food model systems". Journal of the American Oil Chemists' Society 83.3 (2006): 223-230.
9. Davis Paul A and Christine K Iwahashi. "Whole almonds and almond fractions reduce aberrant crypt foci in a rat model of colon carcinogenesis". Cancer Letters 165.1 (2001): 27-33.

10. Hyson Dianne A., et al. "Almonds and almond oil have similar effects on plasma lipids and LDL oxidation in healthy men and women". The Journal of Nutrition 132.4 (2002): 703-707.

11. Venkatachalam Mahesh and Shridhar K Sathe. "Chemical composition of selected edible nut seeds". Journal of Agricultural and Food Chemistry 54.13 (2006): 4705-4714.

12. Moure Andrés., et al. "Antioxidant activity of extracts produced by solvent extraction of almond shells acid hydrolysates". Food Chemistry 101.1 (2007): 193-201.

13. Martinez J M., et al. "Fractionation of residual lignocellulosics by dilute-acid prehydrolysis and alkaline extraction: application to almond shells". Bioresource Technology 52.1 (1995): 59-67.

14. Milbury Paul E., et al. "Determination of flavonoids and phenolics and their distribution in almonds". Journal of Agricultural and Food Chemistry 54.14 (2006): 5027-5033.

15. Pellegrini Nicoletta., et al. "Total antioxidant capacity of spices, dried fruits, nuts, pulses, cereals and sweets consumed in Italy assessed by three different in vitro assays". Molecular Nutrition and Food Research 50.11 (2006): 1030-1038.

16. Donovan Jennifer L., et al. "Phenolic composition and antioxidant activity of prunes and prune juice (Prunus domestica)". Journal of Agricultural and Food Chemistry 46.4 (1998): 1247-1252.

17. Frison-Norrie Suzanne and Peter Sporns. "Identification and quantification of flavonol glycosides in almond seedcoats using MALDI-TOF MS". Journal of Agricultural and Food Chemistry 50.10 (2002): 2782-2787.

18. Harrison $\mathrm{K}$ and L M Were. "Effect of gamma irradiation on total phenolic content yield and antioxidant capacity of almond skin extracts". Food Chemistry 102.3 (2007): 932-937.

19. Chen Chung-Yen., et al. "Flavonoids from almond skins are bioavailable and act synergistically with vitamins $\mathrm{C}$ and $\mathrm{E}$ to 
enhance hamster and human LDL resistance to oxidation". The Journal of Nutrition 135.6 (2005): 1366-1373.

20. Heim Kelly E., et al. "Flavonoid antioxidants: chemistry, metabolism and structure-activity relationships". The Journal of Nutritional Biochemistry 13.10 (2002): 572-584.

21. Takeoka Gary., et al. "Identification of three triterpenoids in almond hulls". Journal of Agricultural and Food Chemistry 48.8 (2000): 3437-3439.

22. Monagas Maria., et al. "Almond (Prunus dulcis (Mill.) DA Webb) skins as a potential source of bioactive polyphenols". Journal of Agricultural and Food Chemistry 55.21 (2007): 8498-8507.

23. Esfahlan Ali Jahanban., et al. "The importance of almond (Prunus amygdalus L.) and its by-products". Food Chemistry 120.2 (2010): 349-360.

24. Pou-Ilinas Juana., et al. "Steam pretreatment of almond shells for xylose production". Carbohydrate Research 207.1 (1990): 126-130.

25. Quesada J., et al. "Ozone oxidation and structural features of an almond shell lignin remaining after furfural manufacture". Holzforschung 56.1 (2002): 32-38.

26. Martinez J M., et al. "Fractionation of residual lignocellulosics by dilute-acid prehydrolysis and alkaline extraction: application to almond shells". Bioresource Technology 52.1 (1995): 59-67.

27. Cruz J M., et al. "Anti-oxidant activity of isolates from acid hydrolysates of Eucalyptus globulus wood". Food Chemistry 90.4 (2005): 503-511.

28. Singh Neha., et al. "Antioxidant activity of processed dry fruits". International Journal of Pharma and Bio Sciences 3.1 (2013): 112-117.

29. Orsavova Jana., et al. "Fatty acids composition of vegetable oils and its contribution to dietary energy intake and dependence of cardiovascular mortality on dietary intake of fatty acids". International Journal of Molecular Sciences 16.6 (2015): 1287112890.

30. Bollin Bradley W., et al. "The phytochemical composition and antioxidant actions of tree nuts". Asia Pacific Journal of Clinical Nutrition 19.1 (2010): 117.
31. Mandalari G., et al. "Characterization of polyphenols, lipids and dietary fibre from almond skins (Amygdalus communis L.)". Journal of Food Composition and Analysis 23.2 (2010): 166174.

32. Font i Forcada., et al. "Association mapping for kernel phytosterol content in almond". Frontiers in plant science 6 (2015): 530

33. Kritchevsky David and Shirley C Chen. "Phytosterols-health benefits and potential concerns: a review". Nutrition Research 25.5 (2005): 413-428.

34. Lee Jihyun., et al. "Quantification of amygdalin in nonbitter, semibitter, and bitter almonds (Prunus dulcis) by UHPLC- (ESI) QqQ MS/MS". Journal of Agricultural and Food Chemistry 61.32 (2013): 7754-7759.

35. Sfahlan Ali Jahanban., et al. "Antioxidants and antiradicals in almond hull and shell (Amygdalus communis L.) as a function of genotype". Food Chemistry 115.2 (2009): 529-533.

36. Takeoka Gary., et al. "Identification of three triterpenoids in almond hulls". Journal of Agricultural and Food Chemistry 48.8 (2000): 3437-3439.

37. Shahidi Fereidoon. "Nutraceuticals and functional foods: whole versus processed foods". Trends in Food Science and Technology 20.9 (2009): 376-387.

38. Özdemir Başak., et al. "Health properties of almond". Journal of Hygienic Engineering and Design 17 (2016): 28-33.

39. Bes-Rastrollo Maira., et al. "Prospective study of nut consumption, long-term weight change, and obesity risk in women". The American Journal of Clinical Nutrition 89.6 (2009): 1913-1919.

40. Holt Susanne HA., et al. "A satiety index of common foods". European Journal of Clinical Nutrition 49.9 (1995): 675-690.

41. Hollis James and Richard Mattes. "Effect of chronic consumption of almonds on body weight in healthy humans". British Journal of Nutrition 98.3 (2007): 651-656.

42. Fraser Gary E., et al. "Effect on body weight of a free 76 kilojoule (320 calorie) daily supplement of almonds for six months". Journal of the American College of Nutrition 21.3 (2002): 275-283. 
43. Jaceldo-Siegl Karen., et al. "Long-term almond supplementation without advice on food replacement induces favourable nutrient modifications to the habitual diets of free-living individuals". British Journal of Nutrition 92.3 (2004): 533-540.

44. Bes-Rastrollo Maira., et al. "Prospective study of nut consumption, long-term weight change, and obesity risk in women". The American Journal of Clinical Nutrition 89.6 (2009): 1913-1919.

45. Rajaram Sujatha and Joan Sabaté. "Nuts, body weight and insulin resistance". British Journal of Nutrition 96.S2 (2006): S79S86.

46. Ternus E M., et al. "Health Benefits of Tree Nuts". Journal of Nutritional Science and Food Technology 9 (2009): 37-65.

47. Jenkins David JA., et al. "The effect of combining plant sterols, soy protein, viscous fibers, and almonds in treating hypercholesterolemia”. Metabolism 52.11 (2003): 1478-1483.

48. Sari M I., et al. "Effect of Prunus dulcis extract against total cholesterol level in mice that given monosodium glutamate". IOP Conference Series: Materials Science and Engineering 288 (2018).

49. Chen Chung-Yen., et al. "A nutrition and health perspective on almonds". Journal of the Science of Food and Agriculture 86.14 (2006): 2245-2250.

50. Timur Taşhan Sermin and Ayşe Kafkasli. "The effect of bitter almond oil and massaging on striae gravidarum in primiparaous women". Journal of Clinical Nursing 21.11-12 (2012): 15701576.

51. Hajhashemi Maryam., et al. "The effect of Aloe vera gel and sweet almond oil on striae gravidarum in nulliparous women". The Journal of Maternal-Fetal and Neonatal Medicine 31.13 (2018): 1703-1708.

52. Korgavkar $\mathrm{K}$ and $\mathrm{F}$ Wang. "Stretch marks during pregnancy: a review of topical prevention". British Journal of Dermatology 172.3 (2015): 606-615.

53. Sultana Yasmin., et al. "Effect of pre-treatment of almond oil on ultraviolet B-induced cutaneous photoaging in mice". Journal of Cosmetic Dermatology 6.1 (2007): 14-19.
54. Sabate Joan and Yen Ang. "Nuts and health outcomes: new epidemiologic evidence". The American Journal of Clinical Nutrition 89.5 (2009): 1643S-1648S.

55. Kelly John H and Joan Sabaté. "Nuts and coronary heart disease: an epidemiological perspective". British Journal of Nutrition 96. S2 (2006): S61-S67.

56. Knoops K T B., et al. "Comparison of three different dietary scores in relation to 10-year mortality in elderly European subjects: the HALE project". European Journal of Clinical Nutrition 60.6 (2006): 746-755.

57. Trichopoulou Antonia and Pagona Lagiou. "Healthy traditional Mediterranean diet: an expression of culture, history, and lifestyle". Nutrition Reviews 55.11 (1997): 383-389.

58. Estruch Ramon., et al. "Effects of a Mediterranean-style diet on cardiovascular risk factors: a randomized trial". Annals of Internal Medicine 145.1 (2006): 1-11.

59. Salas-Salvadó Jordi., et al. "Effect of a Mediterranean diet supplemented with nuts on metabolic syndrome status: one-year results of the PREDIMED randomized trial". Archives of Internal Medicine 168.22 (2008): 2449-2458.

60. Due Anette., et al. "Comparison of the effects on insulin resistance and glucose tolerance of 6-mo high-monounsaturatedfat, low-fat, and control diets". The American Journal of Clinical Nutrition 87.4 (2008): 855-862.

\section{Volume 5 Issue 7 July 2021}

(C) All rights are reserved by Waseem Khalid., et al. 